\title{
PIAS proteins and transcriptional regulation-more than just SUMO E3 ligases?
}

\author{
Andrew D. Sharrocks ${ }^{1}$ \\ Faculty of Life Sciences, University of Manchester, Manchester M13 9PT, United Kingdom
}

The PIAS family of proteins was named based on the identification of the founding member, PIAS3, as a repressor of the activity of the STAT3 transcription factor (Protein Inhibitor of Activated STAT) (Chung et al. 1997). Since then, three additional family membersPIAS1, PIASy, and PIASx - have been identified and are characterized by a high degree of sequence conservation throughout the proteins (for review, see Schmidt and Muller 2003; Shuai and Liu 2005). Additional proteins exist with more limited similarity, including hZIMP7 and hZIMP10 (Sharma et al. 2003; Huang et al. 2005). PIAS proteins have been shown to impact on the function of many different proteins, but a major process on which all these proteins act is in controlling gene transcription. Thus, PIAS proteins can be thought of as transcriptional coregulators. PIAS protein action can either be activating or repressive, although their mechanism of action apparently differs depending on the target gene or interacting transcriptional regulator (for review, see Schmidt and Muller 2003; Shuai and Liu 2005). One major mechanism through which PIAS proteins operate appears to be through the relocalization of transcriptional regulators to different subnuclear compartments. In this issue of Genes \& Development, further weight to this particular mechanism is provided, as PIAS1 is shown to localize the Msx1 homeodomain transcription factor to the nuclear periphery (Lee et al. 2006). This localization is thought to be instrumental in permitting Msx1 to engage with its target promoters and hence cause their repression. However, several other mechanisms of action have been shown for PIAS proteins, although these are not necessarily mutually exclusive with a role in controlling the subcellular localization of target proteins.

\section{PIAS proteins-structural anchors, transcriptional regulators, or SUMO ligases?}

The structure of PIAS proteins provides clues as to their potential functional roles. Indeed, PIAS proteins share a

\footnotetext{
${ }^{1}$ Correspondence.
}

E-MAIL a.d.sharrocks@manchester.ac.uk; FAX 0044-161-275-5082. Article and publication are at http://www.genesdev.org/cgi/doi/10.1101/ gad.1421006. common structure, with five conserved domains/motifs (Fig. 1), although PIASy lacks the C-terminal serine/ threonine-rich domain. The SAP domain is found in numerous proteins that are thought to play a role in chromatin biology, including the scaffold attachment factor SAFA/B and ACINUS, which is involved in chromatin condensation, and it is from these proteins that this acronym is derived (SAFA/B, ACINUS, PIAS). Significantly, this domain is also found in many other transcriptional regulators, including the potent coactivator proteins of the myocardin/MAL family (for review, see Cen et al. 2004). Thus, this suggests a link between transcriptional regulation and chromatin/nuclear matrix attachment. Indeed, the SAP domain of PIASy has been shown to associate with DNA found in matrix attachment regions (MARS) and thereby permit binding to the nuclear matrix (Sachdev et al. 2001). Further links to transcriptional regulation are provided by the observation that PIAS proteins bind to and control the activities of numerous transcriptional regulatory proteins (for review, see Schmidt and Muller 2003; Shuai and Liu 2005). Depending on the PIAS and partner protein involved, either activation or repression of transcription can result. However, the sites of interaction with transcriptional regulators have been mapped throughout the length of the proteins, with no obvious hotspots for interaction.

The other major functional part of PIAS proteins is the SP-RING domain, which is related to the zinc-binding RING fingers and is most similar to those found in a subclass of ubiquitin E3 ligases. This latter observation coupled with their frequent association with SUMOmodified proteins gave rise to the suggestion that PIAS proteins might play an analogous role as E3 ligases for the SUMO modification pathway (Fig. 1). This was, indeed, shown to be the case for proteins such as LEF1, p53, and the androgen receptor (Kahyo et al. 2001; Sachdev et al. 2001; Kotaja et al. 2002; Schmidt and Muller 2002) and has now been shown for many additional proteins (for review, see Shuai and Liu 2005). Recently, studies on the yeast PIAS-like protein Sizl have suggested that the upstream PINIT motif functions together with the RING finger to form a functional module with E3 ligase activity (Takahashi and Kikuchi 2005). PIAS pro- 


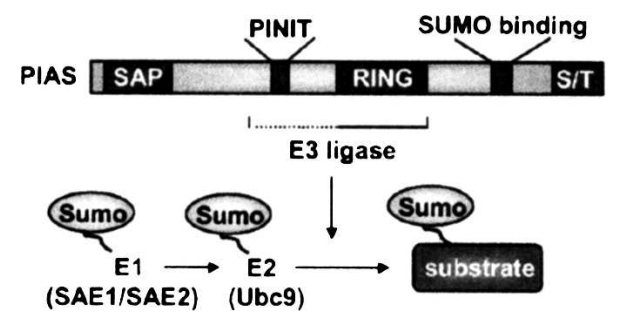

Figure 1. The domain structure of PIAS proteins and their role as SUMO E3 ligases. The minimal E3 ligase domain, including the RING finger, is bracketed. This E3 ligase activity facilitates the transfer of SUMO from Ubc9 (the E2 conjugating enzyme) to substrates such as transcriptional regulators. S/T represents the serine/threonine-rich domain.

teins are also often found to be associated with SUMOmodified substrates (for review, see Schmidt and Muller 2003; Shuai and Liu 2005), further emphasizing their role as potential SUMO ligases. In this mode of function, PIAS proteins are thought to act as adapter proteins that enhance the interactions between the SUMO conjugating enzyme Ubc9 and the substrate proteins. Sumoylation of transcriptional regulators can affect their activities in many ways; however, one particular mechanism is illustrated by SATB2, where PIAS1-mediated sumoylation is important both for imparting repressive properties and for the localization of SATB2 to the nuclear periphery (Fig. 2; Dobreva et al. 2003). This suggests a role for PIAS1 in providing a link between transcription factor subnuclear relocalization and transcriptional repression. Indeed, PIAS1 is further implicated in controlling transcription factor localization at the nuclear periphery through its action toward Msx1 (Lee et al. 2006) (see below for details).

Importantly, however, while sumoylation of transcriptional regulators often leads to changes in their activity (for review, see Gill 2005; Hay 2005), there are a growing number of cases in which the effect of PIAS proteins on substrate function is uncoupled from their SUMO ligase activity against the particular substrate. Thus, it appears that the role of PIAS proteins extends beyond modifying the outputs of the SUMO pathway.

\section{SUMO ligase-independent functions of PIAS proteins}

The Msx1 homeodomain protein represses the expression of muscle-specific genes in undifferentiated cells. Msxl is modified by SUMO, although this modification appears to have little effect on its transcriptional repressive properties (Lee et al. 2006). However, overexpression of PIAS1 enhances the levels of Msx1 sumoylation, and, conversely, knockdown of PIAS1 levels reduces the repressive activity of Msx1, leading to a potential paradox, given that the SUMO modification sites apparently play little role in mediating transcriptional repression. Importantly though, PIAS1 binding to Msxl is required for localizing Msx1 to the nuclear periphery, while the SUMO sites in Msxl are not required for this localization to occur. Thus, it was concluded that it is the lo- calization of Msx1 to the nuclear periphery by PIAS1 binding that is the important determinant of Msx1 repressive activity. In contrast, the SUMO ligase activity of PIAS1 toward Msx1 is apparently unimportant for this process, at least in the context of proteins that are overexpressed.

The authors also demonstrate that several Msx1 target genes are located at the nuclear periphery in undifferentiated muscle cells, where they are inactive, but toward the center of the nucleus in differentiated muscle cells, where the repression is lost (Lee et al. 2006). Thus, it is thought that the recruitment of Msxl to the nuclear periphery by PIAS1 enables it to bind to its target genes and repress them. Indeed, the interaction of Msxl with the MyoD promoter is affected upon PIAS1 depletion. Msx1 binding to a site located $\sim 20 \mathrm{~kb}$ upstream from the transcriptional start site was severely depleted, consistent with the notion that PIAS1 is required for Msxl binding and hence Msxl-mediated transcriptional repression. However, the authors also see enrichment of Msx1 binding to more proximal promoter regions following PIAS1 depletion, suggesting a change in location of promoter occupancy rather than complete loss of Msx1 binding (Lee et al. 2006). Thus, PIAS1 might be directing the binding of Msxl to different parts of the promoter rather than occupancy per se, and this might have different functional consequences. Indeed, this is consistent with the authors' conclusion that PIAS1 controls the DNAbinding specificity of Msxl. However, how this is achieved is currently unclear, and whether the specificity is changed at the nucleotide level, as classically defined, remains to be determined by the comparison of multiple ChIP-verified Msxl sites that are dependent on PIAS1. Together, these results suggest an attractive model whereby PIAS1 recruits Msxl to the nuclear periphery, where it engages with and represses its target promoters (Fig. 2).

The study by Lee et al. (2006) raises several interesting questions. Importantly, it is unclear whether there is any link between the localization of the Msxl target genes and the engagement of the repressive machinery and if so, how is this mediated? It appears that neither Msx1 nor PIAS1 is required for the localization of the Msx1 target genes such as $M y o D$ at the nuclear periphery, suggesting that repression might involve a stepwise mechanism with initial relocalization of the target gene, followed by the engagement of the transcriptional repressor Msx1. It is unclear how the genes are relocalized to the nuclear periphery, but once there, the localization of these genes is not apparently sufficient for their repression. While PIAS1 is clearly needed for the repression of the $M y o D$ promoter contained in a reporter construct, it is not known whether this reporter becomes relocalized to the periphery or whether this is also the case for the endogenous MyoD gene (Lee et al. 2006). Thus, the links between PIAS1-mediated repression and subnuclear relocalization are currently correlative rather than definitive. In a more general context, several genes have now been shown to reside at the nuclear periphery when repressed and to move toward the center of the nucleus 


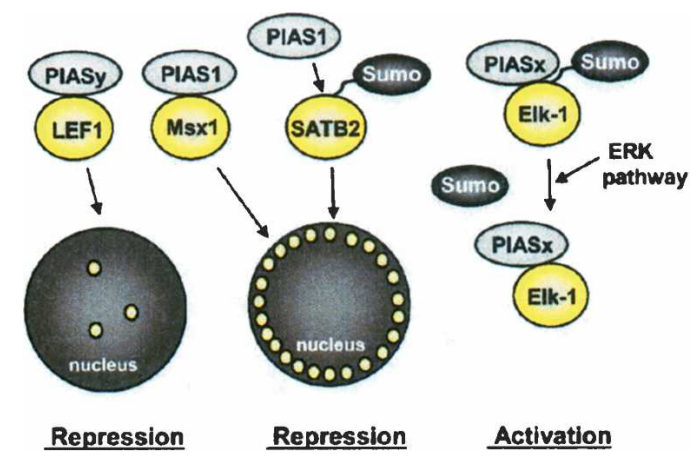

Figure 2. Mechanisms of action of PIAS proteins. PIAS proteins can control subcellular localization through either substrate interaction (e.g., LEF1 and Msx1) or through substrate sumoylation (e.g., SATB2). PIAS proteins can also function in an E3 ligase-independent manner to control transcription factor activity (e.g., Elk-1 where desumoylation is promoted). Subnuclear relocalization and/or substrate sumoylation can lead to either transcriptional repression or activation of target genes for the particular transcriptional regulator.

upon activation, including CFTR (Zink et al. 2004) and Mash1 (Williams et al. 2006), although in other cases, it is apparently the regulatory machinery rather than the gene that changes localization (e.g., the IFNy locus) (Hewitt et al. 2004). Whether PIAS proteins play a role at the nuclear periphery in regulating other genes through promoting repression remains to be determined.

Another interesting question concerns how PIAS1 interaction with Msxl promotes its binding to a particular site in vivo. It would be interesting to investigate if this be recapitulated in vitro with purified components and thereby establish whether PIAS1-mediated enhancement of Msx1-binding specificity is dependent on other proteins and/or subcellular localization. Indeed, PIAS1 might promote interactions with additional DNA-binding proteins that provide enhanced sequence specificity in vivo. However, it is unclear how subnuclear relocalization alone would affect Msx1 binding, unless a promoter subdomain containing the favored Msx1-binding site was brought into close proximity with a region of the nuclear matrix where PIAS1 and Msx1 are located. Importantly, PIAS1 does not always function to promote transcription factor binding to DNA, as knockout studies clearly demonstrate that PIAS1 actually inhibits STAT1 binding to IFN $\gamma$-regulated genes (Liu et al. 2004). In this case, specificity of action is again observed, with PIAS1-mediated inhibition occurring preferentially at "weak" STAT1-binding sites. Thus, PIAS1 can either promote or inhibit transcription factor binding to promoters in a site-selective manner.

Another PIAS protein, PIASy, has been shown to repress the activity of the transcription factor LEF1. In this case, PIASy can recruit LEF1 to a subset of PML nuclear bodies, and it is this recruitment that is thought to be important in repressing the activity of LEF1 (Fig. 2; Sachdev et al. 2001). Thus, subnuclear relocalization of transcriptional regulators by PIAS proteins appears to be a recurring theme, although several different subnuclear localizations appear to be commensurate with repressive activity. Interestingly, PIASy was shown to be able to sumoylate LEF1, and as observed for PIAS1-mediated Msx1 sumoylation, this appears to be inconsequential for transcriptional repression. However, while the role of PIAS-mediated transcription factor sumoylation remains unclear in these cases, it might be that at endogenous levels, it does have an important role, or alternatively, the rather crude reporter assays usually used to assess the role of transcription factor sumoylation maybe inappropriate. Alternatively, sumoylation might either be an inconsequential byproduct that has to be tolerated following PIAS-substrate interactions, and/or which might once have had a function that is now lost. What is clear, however, is that the SUMO E3 ligase activity of PIAS proteins is of differential importance depending on the target substrate and often it is the binding to the substrate that is of more importance.

A further complication in assessing the links between PIAS proteins, subnuclear localization, and transcriptional repression is illustrated by the effect of PIASx on Fli-1 activity (van den Akker et al. 2005). Here PIASx relocalizes Fli-1 to nuclear bodies. However, a SUMO E3 ligase-deficient PIASx protein is unable to do this. Transcriptional repression can, however, be mediated by PIASx mutants that lack E3 ligase activity. Thus, here transcriptional repression and subnuclear localization mediated by PIAS are clearly uncoupled. Furthermore, not all transcriptional regulators are subjected to relocalization by PIAS proteins. A good example of this is the role of PIASx in controlling Elk-1 activity (Yang and Sharrocks 2005). Here, rather than acting as a transcriptional repressor, PIASx promotes transcriptional activation by facilitating Elk-1 activation in response to ERK MAP kinase signaling. The E3 ligase activity of PIASx is apparently not required for this, but conversely, PIASx is actually required for Elk-1 desumoylation and associated HDAC-2 loss, which is a key event in its activation cycle (Fig. 2). How PIASx facilitates this activation process is currently unclear, but this further emphasizes the divergent emerging roles of PIAS proteins in transcriptional regulation, which do not necessarily involve PIAS proteins acting as an E3 ligase for transcriptional regulators.

\section{Perspectives}

It is clear that studies on the mechanisms of action of PIAS proteins are still in their infancy. However, we now know that PIAS proteins can affect the activity of transcriptional regulators in two distinct ways. They can work through acting as SUMO E3 ligases, to enhance substrate sumoylation and hence modify their properties through the addition of SUMO moieties. Alternatively, they can have SUMO-independent effects. In some cases, both modes of action appear to operate. Indeed, PIAS proteins are often associated with SUMO-modified proteins, although it is unclear whether in some cases this is a fortuitous association or has a real functional consequence on SUMO-dependent changes in substrate activ- 
ity. Biologically, studies on knockout mice have so far revealed relatively minor phenotypes for PIAS proteins. PIASx-deficient mice have mild defects in testicular development (Santti et al. 2005). PIAS1 is important in innate immunity through regulating STAT and NFкB signaling (Liu et al. 2004, 2005), whereas loss of PIASy causes modest defects in IFN and Wnt signaling in several cell types including macrophages (Roth et al. 2004; Wong et al. 2004). Whether the lack of dramatic phenotypes is due to functional redundancy is not yet clear, although studies in yeast demonstrate that deletion of the two PIAS-like proteins Siz1 and Siz2 also results in relatively mild effects (Johnson and Gupta 2001). Alternatively, the role of PIAS proteins might be to modulate the thresholds of gene regulation as suggested by the role of PIASx in Elk-1 regulation (Yang and Sharrocks 2005). Here, PIASx is not absolutely required for Elk-1 transactivation capacity and target gene activation, but is required for maximal activation and hence high-amplitude responses to signaling pathways. Similarly, PIASy is apparently needed for high-amplitude gene activation responses to IFN $\gamma$ signaling (Roth et al. 2004). Such subtle modifications in gene regulation may not lead to gross abnormalities, but instead might contribute to the relatively mild phenotypes observed. What recent findings, including the study by Lee et al. (2006), do, however, illustrate is that we are likely to uncover several additional novel modes of action for PIAS proteins and, at this stage, it is wise to consider whether PIAS proteins have further substrate-specific effects rather than extrapolating from the few current examples of how they function. On the flip side, we also need to engage "postgenomic technologies" to gain a fuller picture of how these proteins control gene expression in vivo. Together, these approaches should provide further exciting discoveries on the mechanisms of PIAS protein action and in a more general context, how the subnuclear localization of transcriptional regulators through PIAS protein action might influence the activity of their target genes.

\section{Acknowledgments}

I thank Shen-Hsi Yang and Paul Shore for comments on the manuscript and Wendy Bickmore for helpful discussion. The work on this subject in my laboratory is supported by grants from the Wellcome Trust and the BBSRC.

\section{References}

Cen, B., Selvaraj, A., and Prywes, R. 2004. Myocardin/MKL family of SRF coactivators: Key regulators of immediate early and muscle specific gene expression. J. Cell. Biochem. 93: 74-82.

Chung, C.D., Liao, J., Liu, B., Rao, X., Jay, P., Berta, P., and Shuai, K. 1997. Specific inhibition of Stat3 signal transduction by PIAS3. Science 278: 1803-1805.

Dobreva, G., Dambacher, J., and Grosschedl, R. 2003. SUMO modification of a novel MAR-binding protein, SATB2, modulates immunoglobulin $\mu$ gene expression. Genes \& Dev. 17: 3048-3061.
Gill, G. 2005. Something about SUMO inhibits transcription. Curr. Opin. Genet. Dev. 15: 536-541.

Hay, R.T. 2005. SUMO: A history of modification. Mol. Cell 18: $1-12$.

Hewitt, S.L., High, F.A., Reiner, S.L., Fisher, A.G., and Merkenschlager, M. 2004. Nuclear repositioning marks the selective exclusion of lineage-inappropriate transcription factor loci during $\mathrm{T}$ helper cell differentiation. Eur. J. Immunol. 34: 3604-3613.

Huang, C.Y., Beliakoff, J., Li, X., Lee, J., Li, X., Sharma, M., Lim, B., and Sun, Z. 2005. hZimp7, a novel PIAS-like protein, enhances androgen receptor-mediated transcription and interacts with SWI/SNF-like BAF complexes. Mol. Endocrinol. 19: 2915-2929.

Johnson, E.S. and Gupta, A.A. 2001. An E3-like factor that promotes SUMO conjugation to the yeast septins. Cell 106: 735-744.

Kahyo, T., Nishida, T., and Yasuda, H. 2001. Involvement of PIAS1 in the sumoylation of tumor suppressor p53. Mol. Cell 8: 713-718.

Kotaja, N., Karvonen, U., Janne, O.A., and Palvimo, J.J. 2002. PIAS proteins modulate transcription factors by functioning as SUMO-1 ligases. Mol. Cell. Biol. 22: 5222-5234.

Lee, H., Quinn, J.C., Prasanth, K.V., Swiss, V.A., Economides, K.D., Camacho, M.M., Spector, D.L., and Abate-Shen, C.. 2006. PIAS1 confers DNA-binding specificity on the Msx1 homeoprotein. Genes \& Dev. (this issue).

Liu, B., Mink, S., Wong, K.A., Stein, N., Getman, C., Dempsey, P.W., Wu, H., and Shuai, K. 2004. PIAS1 selectively inhibits interferon-inducible genes and is important in innate immunity. Nat. Immunol. 5: 891-898.

Liu, B., Yang, R., Wong, K.A., Getman, C., Stein, N., Teitell, M.A., Cheng, G., Wu, H., and Shuai, K. 2005. Negative regulation of NF-кB signaling by PIAS1. Mol. Cell. Biol. 25: 1113 1123.

Roth, W., Sustmann, C., Kieslinger, M., Gilmozzi, A., Irmer, D., Kremmer, E., Turck, C., and Grosschedl, R. 2004. PIASydeficient mice display modest defects in IFN and Wnt signaling. J. Immunol. 173: 6189-6199.

Sachdev, S., Bruhn, L., Sieber, H., Pichler, A., Melchior, F., and Grosschedl, R. 2001. PIASy, a nuclear matrix-associated SUMO E3 ligase, represses LEF1 activity by sequestration into nuclear bodies. Genes \& Dev. 15: 3088-3103.

Santti, H., Mikkonen, L., Anand, A., Hirvonen-Santti, S., Toppari, J., Panhuysen, M., Vauti, F., Perera, M., Corte, G., Wurst, W., et al. 2005. Disruption of the murine PIASx gene results in reduced testis weight. J. Mol. Endocrinol. 34: 645654.

Schmidt, D. and Muller, S. 2002. Members of the PIAS family act as SUMO ligases for c-Jun and p53 and repress p53 activity. Proc. Natl. Acad. Sci. 99: 2872-2877.

- 2003. PIAS/SUMO: New partners in transcriptional regulation. Cell. Mol. Life Sci. 60: 2561-2574.

Sharma, M., Li, X., Wang, Y., Zarnegar, M., Huang, C.Y., Palvimo, J.J., Lim, B., and Sun, Z. 2003. hZimp10 is an androgen receptor co-activator and forms a complex with SUMO-1 at replication foci. $E M B O$ J. 22: 6101-6114.

Shuai, K. and Liu, B. 2005. Regulation of gene-activation pathways by PIAS proteins in the immune system. Nat. Rev. Immunol. 5: 593-605.

Takahashi, Y. and Kikuchi, Y. 2005. Yeast PIAS-type U111/Siz1 is composed of SUMO ligase and regulatory domains. J. Biol. Chem. 280: 35822-35828.

van den Akker, E., Ano, S., Shih, H.M., Wang, L.C., Pironin, M., Palvimo, J.J., Kotaja, N., Kirsh, O., Dejean, A., and Ghysdael, J. 2005. FLI-1 functionally interacts with PIASx $\alpha$, a member 
Sharrocks

of the PIAS E3 SUMO ligase family. J. Biol. Chem. 280: 38035-38046.

Williams, R.R., Azuara, V., Perry, P., Sauer, S., Dvorkina, M., Jorgensen, H., Roix, J., McQueen, P., Misteli, T., Merkenschlager, M., et al. 2006. Neural induction promotes largescale chromatin reorganisation of the Mash1 locus. J. Cell Sci. 119: 132-140.

Wong, K.A., Kim, R., Christofk, H., Gao, J., Lawson, G., and Wu, H. 2004. Protein inhibitor of activated STAT Y (PIASy) and a splice variant lacking exon 6 enhance sumoylation but are not essential for embryogenesis and adult life. Mol. Cell. Biol. 24: 5577-5586.

Yang, S.H. and Sharrocks, A.D. 2005. PIASx acts as an Elk-1 coactivator by facilitating derepression. EMBO I. 24: 21612171.

Zink, D., Amaral, M.D., Englmann, A., Lang, S., Clarke, L.A., Rudolph, C., Alt, F., Luther, K., Braz, C., Sadoni, N., et al. 2004. Transcription-dependent spatial arrangements of CFTR and adjacent genes in human cell nuclei. J. Cell Biol. 166: 815-825. 


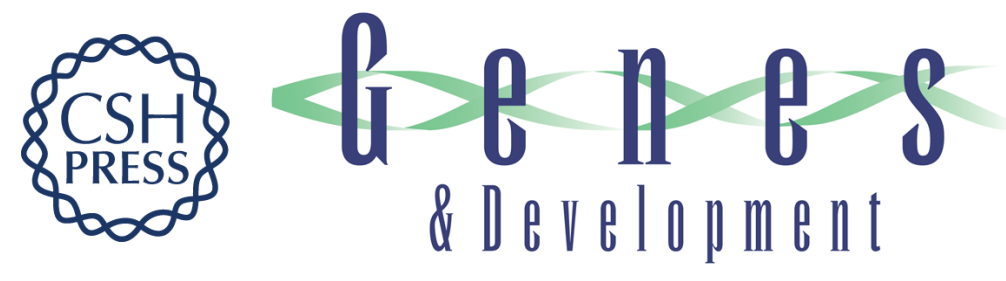

\section{PIAS proteins and transcriptional regulation--more than just SUMO E3 ligases?}

Andrew D. Sharrocks

Genes Dev. 2006, 20:

Access the most recent version at doi:10.1101/gad.1421006

\section{Related Content \\ PIAS1 confers DNA-binding specificity on the Msx1 homeoprotein \\ Hansol Lee, John C. Quinn, Kannanganattu V. Prasanth, et al. \\ Genes Dev. UNKNOWN , 2006 20: 784-794 \\ References This article cites 25 articles, 13 of which can be accessed free at: http://genesdev.cshlp.org/content/20/7/754.full.html\#ref-list-1 \\ Articles cited in: \\ http://genesdev.cshlp.org/content/20/7/754.full.html\#related-urls \\ License
Email Alerting
Service
Receive free email alerts when new articles cite this article - sign up in the box at the top right corner of the article or click here.

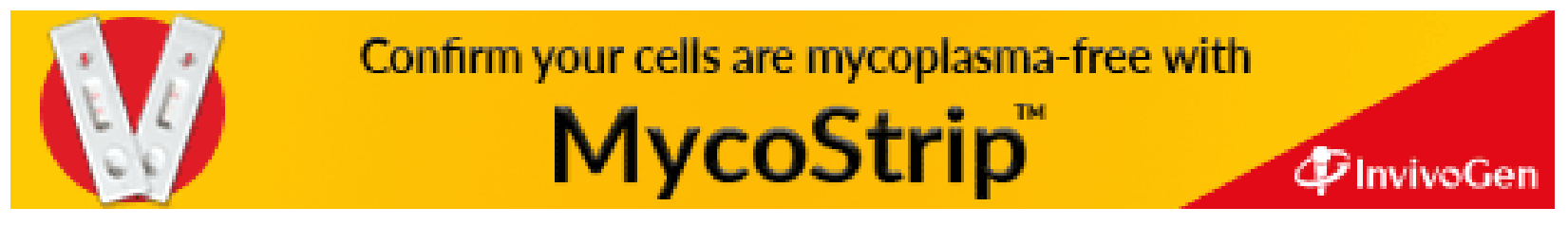

